\title{
A Parallel Algorithm For Anonymizing Large-scale Trajectory Data
}

\author{
KATRINA WARD, Missouri University of Science and Technology, USA \\ DAN LIN, University of Missouri, USA \\ SANJAY MADRIA, Missouri University of Science and Technology, USA
}

\begin{abstract}
With the proliferation of location-based services enabled by a large number of mobile devices and applications, the quantity of location data, such as trajectories collected by service providers, is gigantic. If these datasets could be published, then they would be valuable assets to various service providers to explore business opportunities, to study commuter behavior for better transport management, which in turn benefits the general public for day-to-day commuting. However, there are two major concerns that considerably limit the availability and the usage of these trajectory datasets. The first is the threat to individual privacy, as users' trajectories may be misused to discover sensitive information, such as home locations, their children's school locations, or social information like habits or relationships. The other concern is the ability to analyze the exabytes of location data in a timely manner. Although there have been trajectory anonymization approaches proposed in the past to mitigate privacy concerns. None of these prior works address the scalability issue, since it is a newly occurring problem brought by the significantly increasing adoption of location-based services. In this article, we conquer these two challenges by designing a novel parallel trajectory anonymization algorithm that achieves scalability, strong privacy protection, and high utility rate of the anonymized trajectory datasets. We have conducted extensive experiments using MapReduce and Spark on real maps with different topologies, and our results prove both effectiveness and efficiency when compared with the centralized approaches.
\end{abstract}

CCS Concepts: • Security and privacy $\rightarrow$ Social aspects of security and privacy; Privacy protections; - Computing methodologies $\rightarrow$ MapReduce algorithms; Massively parallel algorithms;

Additional Key Words and Phrases: Trajectory anonymization, Large-scale, Divide-and-conquer, MapReduce

ACM Reference format:

Katrina Ward, Dan Lin, and Sanjay Madria. 2020. A Parallel Algorithm For Anonymizing Large-scale Trajectory Data. ACM/IMS Trans. Data Sci. 1, 1, Article 5 (January 2020), 26 pages.

https://doi.org/10.1145/3368639

This work was funded by the National Science Foundation (Grant No. NSF-DGE-1914771) and Department of Education (Grant No. P200A120110).

Authors' addresses: K. Ward, Missouri University of Science and Technology, 500 West 15th street, Rolla, MO, 65409, USA; email: kjw26b@mst.edu; D. Lin (corresponding author), University of Missouri, 126 Naka Hall, Columbia, MO, 65211, USA; email: lindan@missouri.edu; S. Madria, Missouri University of Science and Technology, 500 West 15th street, Rolla, MO, 65409, USA; email: madrias@mst.edu.

Permission to make digital or hard copies of all or part of this work for personal or classroom use is granted without fee provided that copies are not made or distributed for profit or commercial advantage and that copies bear this notice and the full citation on the first page. Copyrights for components of this work owned by others than ACM must be honored. Abstracting with credit is permitted. To copy otherwise, or republish, to post on servers or to redistribute to lists, requires prior specific permission and/or a fee. Request permissions from permissions@acm.org.

(C) 2020 Association for Computing Machinery.

2577-3224/2020/01-ART5 \$15.00

https://doi.org/10.1145/3368639

ACM/IMS Transactions on Data Science, Vol. 1, No. 1, Article 5. Publication date: January 2020. 


\section{INTRODUCTION}

One of the fastest growing trends in mobile technology is the use of location-based services for applications such as social networking, vehicle tracking, and targeted advertising. As reported by Census, more than 471,000 people commute into Los Angeles county everyday, which results in more than 10 million trajectories each month [4]. According to Cisco, global mobile data traffic has reached 7.2 exabytes a month and is increasing rapidly [28]. In 2013, 526 million mobile devices were added to cellular and WiFi networks, including a large increase in data usage for locationbased mobile applications. Currently, $74 \%$ of adults use their smart phones to get directions and other information based on their current location. Thirty percent of adults with an account on social media sites say they have at least one of those accounts include their current location in their posts [34]. Even if we ignore hand-held devices, as many as $96 \%$ of cars produced in 2013 are built with event recorders that include GPS. As a result, a huge amount of location information has been collected and stored for analytics.

These collected location data have great potential for statistical usage in various applications, such as traffic congestion prevention, infrastructure and evacuation planning, analysis of social behavior, advertising campaigns, and control of spread of diseases. While the benefits provided by location datasets are indisputable, many challenges remain to be addressed to actually realize the benefits. One critical challenge is how to ease customers' worries on location privacy [18] when they are told their location data have been collected and may be used for analysis. A politician in Germany went to court to find out how much and what kind of data was collected by his cell phone company. The results were staggering. In just a six-month period, his coordinates were recorded and stored 35,000 times [2]. Without a strong privacy guarantee, cautious customers will soon be reluctant to subscribe to location-based services. To address the privacy concern, several methods have been proposed, such as k-anonymization, spatial-cloaking, and data transformation $[1-3,8-11,13,17,20,22-24]$. Unfortunately, none of the prior works addresses the scalability issue, which is a newly occurring problem brought by the information explosion, i.e., the current need of dealing with exabytes of location data compared to only millions of data in the past.

In this article, we propose a novel parallel trajectory anonymization algorithm under the map-reduce [7] programming paradigm, called MELT, which encloses the following four features: Mapreduce-based, Efficient, Large-scale Trajectories, and "melting" the trajectories for anonymization to preserve their privacy. We present our algorithms by following the MapReduce programming paradigm, since it clearly exemplifies the divide-and-conquer spirit, which is heavily used in our proposed parallel algorithm.

It is not a trivial task to convert existing centralized anonymization approaches to a parallel version. To better understand the challenge, let us look at the following naive solutions. A common approach for handling a large-scale dataset is to partition the big trajectory dataset into many small datasets and then anonymize individual small datasets in parallel using the existing centralized approach. For dataset partitioning, there are two straightforward methods. One is to partition the road map vertically (or horizontally) into equal-width stripes, and the other is to partition the map into grids of equal-size cells. Then, anonymize trajectories that fall in each partition separately. However, such partitioning of trajectory datasets can easily split a single trajectory into multiple segments as shown in Figure 1(a), where solid lines indicate trajectories and dashed lines indicate partitioning. As a result, groups of popular trajectories that are across multiple partitions will be treated separately, which could severely lower the utility of the anonymized data. This is because some trajectories will be anonymized segment by segment rather than being considered as a whole. The anonymization results may contain disconnected or shorter trajectories, which would have lower data utility rate compared to that produced by the original centralized approach that directly 


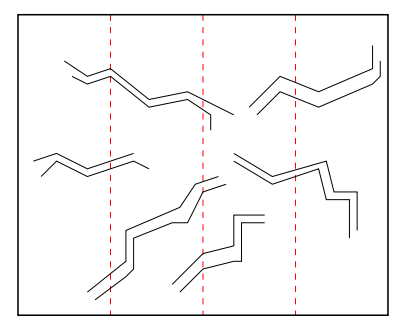

(a) Vertical Partitioning

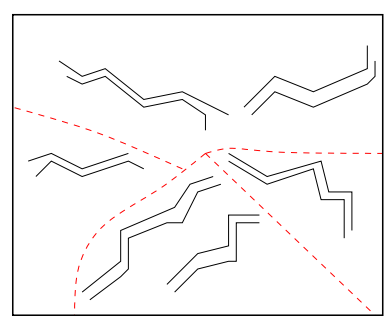

(b) Ideal Partitioning

Fig. 1. (a) Naive map-partitioning approaches break up trajectories into small segments, which will lower data utility. (b) Ideal partitioning to keep trajectories whole.

works on the entire dataset. Moreover, anonymizing partial trajectories can introduce additional privacy breach, called inference-route problems as elaborated later.

An ideal case is to have the map partitions match the natural clusters of trajectories as shown in Figure 1(b), so that the quality of the anonymization results will be as close as that obtained from the centralized approach and original data. To achieve this, one may think of using existing trajectory clustering algorithms [15] to get the map partitions. However, this would not work either, because clustering algorithms typically requires scanning the dataset multiple times. Multiple scanning of exabytes of trajectory data is very time and resource consuming, and thus it may not be practical. Instead, we expect to scan the dataset at most once.

To overcome the aforementioned challenges, in this article, our proposed MELT algorithm makes the following new contributions:

- We propose a dynamic data partitioning algorithm that automatically adapts to the distribution of trajectories and partitions the map to capture the natural clusters of trajectories.

- We propose three rounds of map-reduce processes that fully parallelize the conventional trajectory anonymization process within a single scan of the dataset.

- We ensure that the anonymization results achieve $k$-anonymity, inference free, and high data utility rate. That is, any trajectory in the published anonymization results will have at least $k-1$ other identical trajectories, and no further information can be inferred from analyzing and comparing trajectories in the anonymization results.

- We analyze the trade-off between privacy and data utility and also conduct extensive experiments on real road maps using Hadoop and Spark. The experimental results demonstrate that our approach is capable of efficiently handling large-scale datasets that cannot be processed by the centralized approach.

The rest of the article is organized as follows. Section 2 reviews the related works. Section 3 formally presents our problem statement. Section 4 elaborates the detailed algorithms of our proposed MELT. Section 5 analyzes the trade-off between privacy and data utility. Section 6 reports the experimental study. Finally, Section 7 concludes the article.

\section{RELATED WORK}

In this section, we first review conventional centralized approaches on privacy-preserving location data publishing. Then, we introduce some recent works on parallel processing of large-scale location data. 


\subsection{Privacy Preserving Trajectory Publishing}

There have been many centralized approaches for trajectory anonymization. Most of them $[9,10$, 13, 20, 22-24] output anonymized trajectories in the form of cloaking regions or centers of clusters. For example, in Reference [10], the spatial-temporal cloaking technique is applied to generate cloaking regions covering segments of trajectories. In Reference [1], Abul et al. consider a trajectory as a cylindrical volume where the radius represents the location imprecision. They then perturb and cluster trajectories with overlapping volumes to ensure that each released trajectory volume encloses at least $k-1$ other trajectories to achieve $k$-anonymity. Similarly, in Reference [20], each trajectory is an ordered set of spatio-temporal 3D volumes. In Reference [19], Monreale et al. cluster trajectories and transform them into a sequence of centroids of Voronoi cells. In these approaches, the anonymized trajectories do not follow the road network constraints. They can be located even in the middle of two parallel roads, and hence, are not beneficial for traffic analysis on individual roads, which our approach aims to achieve. Also, GPS systems are becoming more and more accurate. A cellphone can tell a person's location within a few feet, making methods such as these unusable in practice.

In Reference [5], trajectories are clustered based on a distance function and then a location time triple in an anonymized trajectory is replaced by an existing triple with close proximity in the original trajectory to satisfy k-anonymity. However, two triples, though close in proximity, may belong to two different roads making it easy for the adversary to identify fake trajectories given that the road map is publicly known. In Reference [1], Abul et al. used a coarsening strategy that removes one or more spatial points in a trajectory to achieve anonymization. An anonymized trajectory may contain disconnected paths. This is different from our approach, which preserves continuous trajectories based on road-network information. Similarly, in Reference [3], Chen et al. adopt a greedy algorithm to suppress locations in the trajectories to achieve anonymity. However, using suppression alone may decrease the utility of the anonymization results. They did not provide any experimental study to prove the effectiveness of the approach. Unlike the previous works, which are based on the similarity of trajectories, Yarovoy et al. [31] group trajectories based on so-called quasi-identifiers, which are hard to be selected in practice. Anonymization using these quasi-identifiers is nearly impossible as knowledge of more than one of these can lead an adversary to infer information about a user.

Rather than representing trajectories as a set of coordinates, some works [19] represent trajectories as landmarks or locations of interests. However, these kind of trajectories provide mainly moving patterns rather than real trajectories as considered in our work. In addition, a few works $[1,3]$ make the assumption that attackers have certain prior knowledge and take such prior knowledge as input for anonymization, while our anonymization is more general and does not need such assumptions.

Finally, we would like to discuss the more closely related work [11, 21, 23], which generate anonymized trajectories following the road-networks. In Reference [21], Pensa et al. proposed a prefix-tree-based anonymization algorithm, which guarantees $k$-anonymity of the published trajectories in a way that no trajectories with support less than $k$ will be published. Later, Gurung et al. [11] identified a so-called inference-route problem in the anonymization results produced by Reference [21], and they proposed an improved anonymization algorithm that achieves stronger privacy guarantee. Our proposed parallel trajectory anonymization algorithm is developed based on this latest centralized trajectory anonymization strategy [11], while addressing new challenges raised by the scalability and the MapReduce technique. In References [22-24], Poulis et al. have proposed a cluster-based anonymization that is similar, but they do not address scalability and also suffer from the inference-route problem as defined in Reference [11]. Most recently, He et al. 
[14] proposed a method to protect user privacy by not publishing the real data generated by users. Instead, they take samples of real datasets and then generate a synthetic trajectory dataset based on information from the real data, probabilities, and noise added. While this does protect user privacy, it causes a loss of utility, which the authors do not analyze. Additionally, there is still the inference-route problem later discussed in Section 3. Our solution is proven to retain utility in large scale, while avoiding the inference-route problem. Additionally, MELT allows the user to select the level of privacy, $k$, and thus adjust the trade-off between privacy and utility. This is something not allowed in Reference [14].

In addition, an overview of our work was introduced briefly in Reference [30]. In this article, we have made the following new contributions: our new algorithm allows each user to define his/her own privacy requirement; we conducted detailed analysis regarding the trade-off between privacy and utility as well as complexity; we added a detailed description and examples for the proposed algorithms; we implemented our work in both Hadoop and Spark.

\subsection{Processing Location Data In Parallel}

Many works [32] have been proposed to utilize map-reduce to perform data mining, pattern classifications, document retrieval, and so on, in large-scale datasets. However, there are very few works on large-scale trajectory data processing. Yang et al. [12] proposed a method called TRUSTER for query processing over trajectory data using MapReduce. In References [16, 25], MapReduce-based algorithms have been proposed to handle trajectory matching. Eldawy and Mokbel [6] proposed SpatialHadoop as an extension to MapReduce to provide support for spatial data management. However, to the best of our knowledge, while these works address indexing and querying trajectories very well, none of the existing works address the issue of anonymizing a large volume of trajectory data.

Aside from Hadoop, there are other parallel approaches. In the most recent work [26, 27], Shang et al. find similar trajectories and group them together in parallel. Compared to their work, which requires multiple passes over the data, our approach is capable of scanning the dataset only once and therefore further reducing the computation time and increasing utility.

\section{PROBLEM STATEMENT}

In this work, we aim to anonymize large-scale trajectory datasets in parallel while maintaining high data utility.

\subsection{Data Representation}

Following the same setting as that in Reference [11], the road network is modeled as a directed graph, where each edge corresponds to a road with objects moving in one direction, and each node represents an intersection. Raw data collected by location-based applications contains their user information as a three-tuple $\langle I D, l o c, t\rangle$, where $I D$ is the user ID the user's location, loc, which corresponds to a GPS coordinate at an intersection, at timestamp $t$, respectively. Each road segment between intersections is assigned a road id $r_{i}$. The anonymization is carried out on the set of trajectories (Definition 1) within the same time interval $t_{\text {int }}$ to preserve the time relationship among trajectories.

Definition 1 (Trajectory). User $u$ 's trajectory is represented as $\operatorname{Tr}_{u}=\left\{r_{1}, r_{2}, \ldots, r_{n}\right\}$, where $r_{1}, \ldots, r_{n}$ are IDs of roads visited by $u$ in sequential time order.

After anonymization, the output dataset contains information in the form of $\left\langle r_{1} d i r_{1}, r_{2} d i r_{2} \ldots\right.$, $r_{n} d i r_{n}$, support $\rangle$, where $r_{i}$ is a road id in the representative trajectory of nodes visited by users, $d i r_{i}$ is the direction the user traveled on $r_{i}$, and support is the number of users who have traveled the 


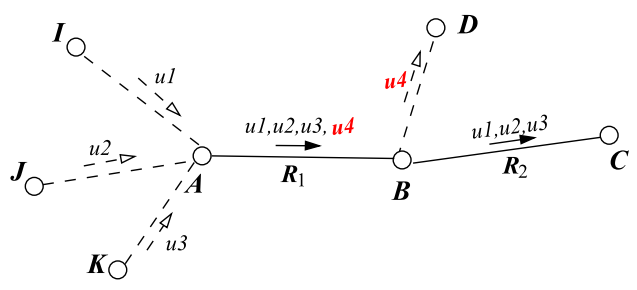

Fig. 2. An Example of the Inference-Route Problem. In this figure, user $u 4$ 's trajectory can be inferred.

entire representative trajectory. Such representation is sufficient to derive trajectories or trafficflow information.

\subsection{Privacy Definition}

We aim to achieve strict $k$-anonymity. That is, no trajectory will be published unless at least $k-1$ other trajectories are identical. The intuition is that if $k$ is sufficiently high, it is impossible to identify an individual user among $k$ identical users.

Definition 2 (Strict $k$-anonymity Over Trajectories). Let $\operatorname{Tr} j$ be a trajectory. We say $\operatorname{Tr} j$ satisfies strict $k$-anonymity if Support(Trj) is no less than $k$, where Support(Trj) is the number of identical trajectories matching $\operatorname{Tr} j$.

Trajectories that satisfy strict $k$-anonymity do not contain any inference-route problem [11]. The notions regarding the inference-route problem are given below.

Definition 3 (Road Frequency). Let $W$ be a time interval, and let $k$ be a threshold. We say a road is a frequent road if the number of objects moving along one direction on this road is no less than $k$ within time interval $W$. We call the number of moving objects as the frequency of the road.

Definition 4 (Inference Route). Let $\Upsilon$ be an intersection of roads $r_{1}, \ldots, r_{m}$, and let $U_{i}^{+}, U_{i}^{-}$be the sets of objects moving toward and outward $\Upsilon$ on road $r_{i}(1 \leq i \leq m)$ during $W$, respectively. If $\exists$ $U_{i}^{+}, U_{j}^{-},\left|U_{i}^{+}\right| \geq k,\left|U_{j}^{-}\right| \geq k$, and $\left(0<\left|U_{i}^{+}-U_{j}^{-}\right|<k\right.$ or $\left.0<\left|U_{j}^{-}-U_{i}^{+}\right|<k\right)$, then we say $\Upsilon$ has an inference-route problem.

In the above definition, the constraints $\left|U_{i}^{+}\right| \geq k,\left|U_{j}^{-}\right| \geq k$ ensure that only frequent road segments are considered, and $\left(0<\left|U_{i}^{+}-U_{j}^{-}\right|<k\right.$ or $\left.0<\left|U_{j}^{-}-U_{i}^{+}\right|<k\right)$ check if there is a chance for a road segment to be inferred. To have a better understanding, let us consider a toy example in Figure 2, which shows four users leaving their homes $(I, J, K, A)$ for work. Let $k$ be 3, which means a trajectory can be published if at least three users have this trajectory. If trajectories are anonymized segment by segment due to map partitioning (illustrated by dashed dotted lines), then the anonymization output will contain two roads $r_{1}$ and $r_{2}$ with supports 4 and 3, respectively, and has the following the inference-route problem. Specifically, if an adversary observes that Alice passes by road $r_{1}$ and $r_{3}$ every weekday, then Bob can infer that $u_{4}$ is Alice's ID from the published anonymized trajectory dataset, because Alice is the only one who may enter road $r_{3}$ by comparing the set of anonymous IDs in the published road segment. Using this ID, the adversary would be able to track Alice's other movement. According to Definition 4 , node $B$ is an intersection of three roads. On road $r_{1}, U_{r_{1}}^{+}=\left\{u_{1}, u_{2}, u_{3}, u_{4}\right\}$; on road $r_{2}, U_{r_{2}}^{-}=\left\{u_{1}, u_{2}, u_{3}\right\}$. Since $U_{r_{1}}^{+}-U_{r_{2}}^{-}=\left\{u_{4}\right\},\left|U_{r_{1}}^{+}-U_{r_{2}}^{-}\right|=1<k$, node $B$ has an inference-route problem. In general, given a threshold $k$, if an adversary can link any anonymous ID to a particular user with probability greater than $\frac{1}{k}$ by using the above method, then we say there is an inference-route problem. 


\subsection{Performance Metrics}

Our proposed trajectory anonymization approach will be evaluated in terms of three factors: (i) privacy guarantee (discussed in the previous section), (ii) efficiency (CPU time), and (iii) data utility rate.

The data utility of the anonymized trajectory dataset will be evaluated using the following two commonly accepted metrics: Precision and Recall. Intuitively, the less difference between the anonymized dataset and the original dataset, the better quality the anonymized dataset is. It is clear that some error will be introduced due to the divide-and-conquer technique inherent to a distributed approach. To gauge the utility of the final dataset, we compare the results from our approach to the original dataset. Recall will allow us to see how much data is lost by splitting the data across multiple nodes that are unable to share information with one another. Precision will tell us how similar our results match those of the original data, thereby showing how much error is introduced. The calculations for these are as follows:

$$
\begin{gathered}
\text { Precision }=\frac{\text { Pairs_Matching_Traj }}{\text { Traj_Output_By_MapReduce }} \\
\text { Recall }=\frac{\text { Pairs_Matching_Traj }}{\text { Pairs_Matching_Traj }+ \text { Missing_Traj }}
\end{gathered}
$$

In the above equations, precision is calculated by comparing the anonymized trajectories obtained from our distributed approach using MELT to the original dataset. Specifically, for each trajectory obtained by our algorithm, we look for the most similar trajectory from the original data set, i.e., the trajectory with the largest number of common nodes and compare the support from each approach. If the identified pair of trajectories are identical, then it means we were able to find the same cluster and that trajectories were grouped together correctly and error was not introduced. Then, the pair are removed from the data sets when searching for the next pair of identical trajectories. In the equation for Recall, Missing ${ }_{T}$ raj refers to the number of trajectories that the centralized approach [11] found that our algorithm did not. This is due to similar trajectories not being partitioned to the same region of the map or similar trajectories becoming dissimilar if broken up across multiple regions, and so they were removed for not meeting our $k$ threshold. The higher the precision and recall, the better the accuracy of our approach and the less error has been introduced.

\section{THE PROPOSED MELT}

In this section, we first present an overview of our proposed MELT algorithm, and then elaborate the detailed steps.

\subsection{An Overview of MELT}

Figure 3 illustrates the overall data flow in the MELT system. The proposed MELT system consists of two major phases: (i) map partitioning; and (ii) divide-and-conquer-based trajectory anonymization. The second phase is further divided into three rounds of processes to ensure the maximum parallelism of the anonymization steps, which are Trajectory Assignment, Pre-Classification, and Melt.

- Map partitioning: At the beginning, we divide the road map into multiple regions with the goal of keeping as many similar trajectories as possible in the same region. A desirable partitioning (as shown in Figure 3) will prevent long frequent traffic flows from being separated or wrongly pruned, and retain as much of the original data as possible to maximize the data 


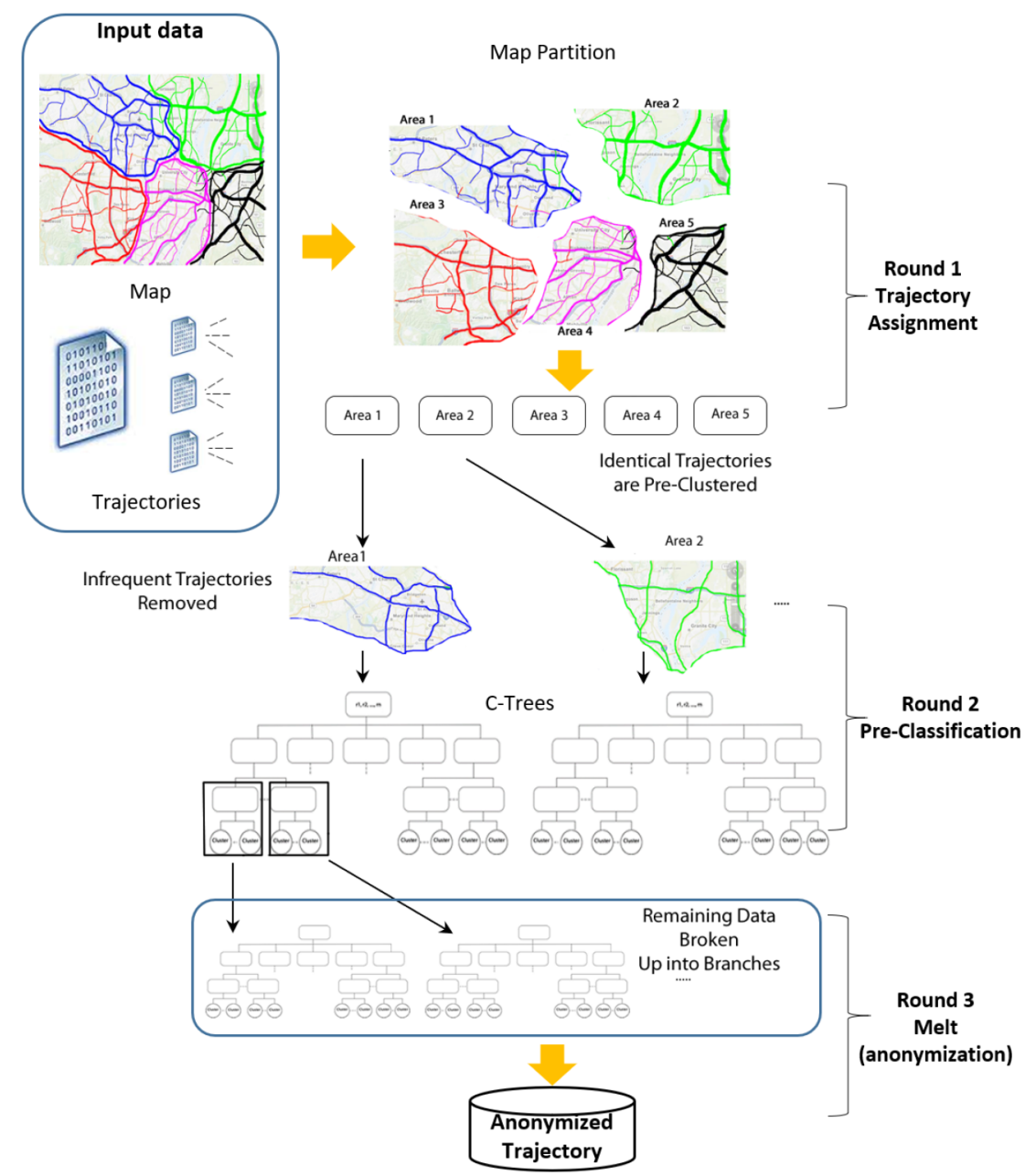

Fig. 3. An overall representation of the architecture of our approach.

utility after the anonymization. However, the map partitioning is an extremely challenging task, because it is done before seeing all the actual data.

- map-reduce-based trajectory anonymization: We design three rounds of map-reduce processes to fully parallelize the trajectory anonymization process. The Trajectory Assignment round takes the map-partitioning results as input, assigns original trajectories to the corresponding regions, and calculates the supports of the same trajectories in each region. The Pre-classification round removes the roads that could cause the inference-route problem, and then organizes the remaining trajectories into a tree structure based on their similarity. Finally, the Melt round conducts the fine-grained anonymization on the sub-trees obtained from the previous round.

\subsection{Road Map Partitioning}

The first step in our approach is to partition the map into regions that meet the following conditions: (i) each region contains an approximately equal number of trajectories, so that the workload 
can be balanced for the worker nodes during the parallel anonymization phase; (ii) each region contains trajectories that are as similar as possible to one another so that the anonymization results will preserve high data utility rate as previously discussed. However, achieving these two goals is very challenging, since we do not want to scan the entire location dataset to determine the regions, which would otherwise be too time consuming. Therefore, we propose an adaptive map-partitioning algorithm (shown in Algorithm 1) that partitions the map based on the concept of the traffic flow. Though we could also parallel this step in our approach, we note that even for the largest city map, we are able to partition it in an order of milliseconds. Thus, we chose not to complicate this step by making it parallel.

To obtain map partitions adapted to different traffic flow datasets, the key idea of our approach is to identify hot spots on the road-networks using a small sample dataset, and then expand them to reconstruct possible traffic flow that forms the sub-regions. To determine a good sampling rate, we conducted extensive tests on various datasets and found that any sample above $30 \%$ preserves nearly the same accuracy compared to using the entire dataset. From the sample dataset, we extract hot spots as follows. Hot spots are popular road intersections. Observe that trajectories tend to frequently pass by some hot spots such as commercial centers, major road-highway intersections, and intersections in between residential areas. Based on this observation, we aim to identify hot spots as hubs in each map partition. Specifically, we compute node frequency (i.e., the sum of trajectories from the sample data set passing by the node/intersection) and road frequency (Definition 3) from the sample trajectories. We sort all the nodes in a descending order of its frequency.

Then, we start a depth-first road expansion from the most frequent nodes. It is worth noting that we can parallel this road expansion process by starting the expansion simultaneously from the top $m$ frequent nodes, which are at a certain distance from each other. Given a starting node $\left(n_{0}\right)$, the road segments connected to $n_{0}$ will be considered in a descending order of their road frequency. At the first expansion, the road $\left(r_{0}\right)$ with the highest road frequency will be selected. After that, the road $r_{1}$ with the most similar road frequency to $r_{0}$ will be selected, and then $r_{2}$ with the most similar road frequency to $r_{1}$. In general, each time we select the subsequent road segment that minimizes $\Delta=f\left(r_{i}\right)-f\left(r_{i-1}\right)$, where $f\left(r_{i}\right)$ and $f\left(r_{i-1}\right)$ denote the frequency of the road $r_{i}$ and its previous road $r_{i-1}$. In this way, we project possible traffic flow. There are three stopping criteria for the expansion, of which only one needs to be met: (i) the length between the hot spot and the current node is equal to or greater than the average length of the trajectories in the data set; (ii) the road-frequency difference $(\Delta)$ exceeds the threshold $\rho=\frac{\max f\left(r_{i}\right)}{d_{r_{i}}-1}$, where $d_{r_{i}}$ is the degree of the node (the number of the roads connected to this intersection). The threshold computes the case when an incoming traffic flow is equally distributed among outgoing roads; (iii) the current area being calculated exceeds $\frac{\text { total_map_area }}{\text { num_regions }}$. Then, the next round of expansion will start again from $n_{0}$ to see if more traffic flow can be identified. If not, then we move to the node with the next highest frequency that is not already included in the previous road expansion, and continue the same road expansion as we did for $n_{0}$. The roads associated with the same hot spot form a region.

After we visit all the hot spots, there may still be some roads that are not yet included in any region. These roads are usually sparsely distributed and mixed in the roads that have already been classified. In this case, we conduct a quick breath-first search starting from unvisited nodes found in the road map and find the closest region for each unvisited node. If two regions are of the same distance to the unvisited node, then the region with the less difference in road frequency will be selected. At the end of our map partitioning, every road will be matched to a region. 


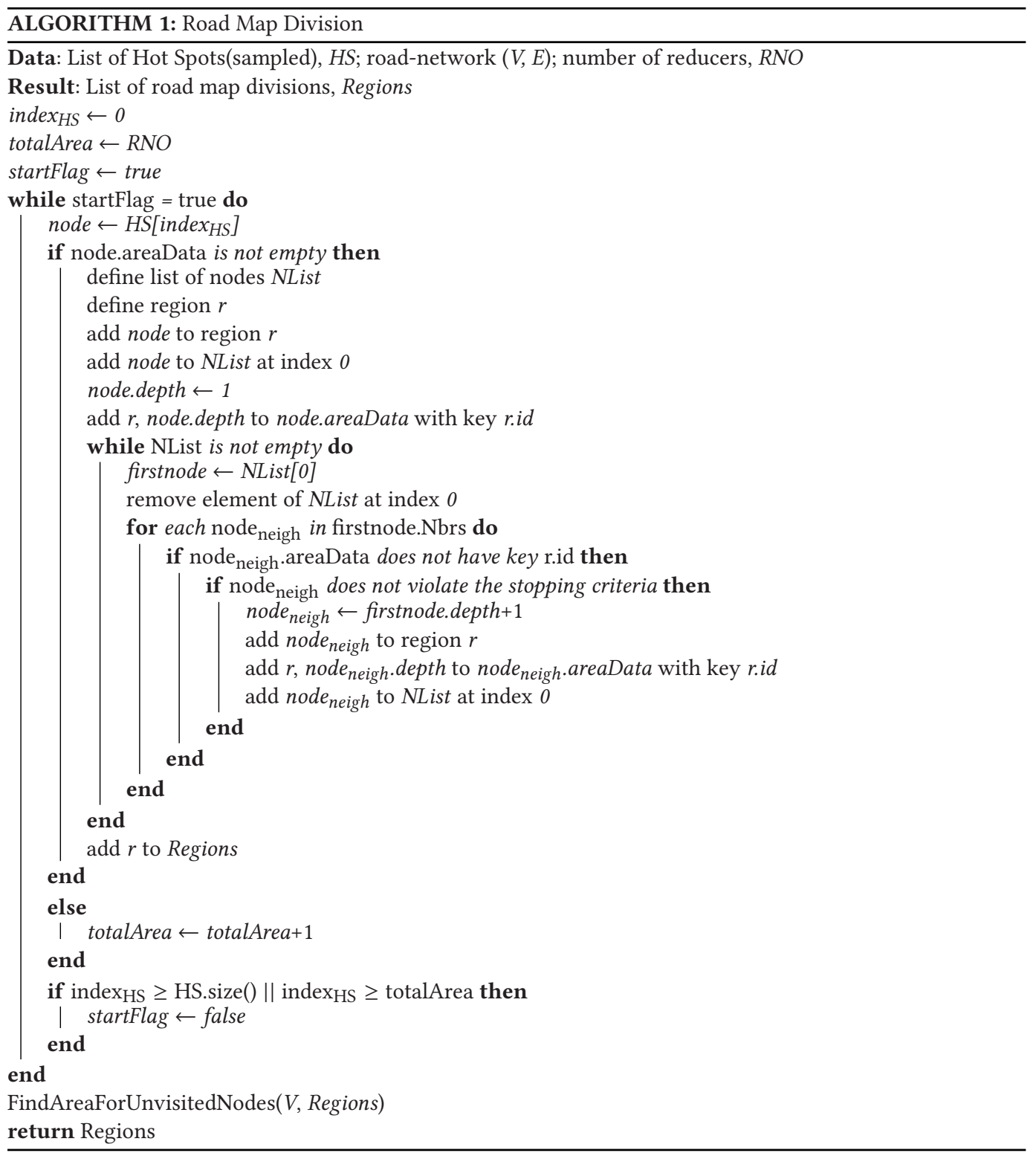

\subsection{Parallel Trajectory Anonymization}

After the map partitioning, we now proceed to discuss how to conduct trajectory anonymization in parallel. A straightforward way to convert the centralized anonymization algorithm to a parallel version is to directly execute the centralized algorithm for the sub-dataset in each region obtained from the map partitioning. However, this straightforward approach does not fully parallel many functions in the anonymization process, is still unable to process the volume of data, and is much less efficient than our proposed approach, as we will soon observe in the experiments.

To parallelize the entire anonymization process as much as possible, we study the centralized algorithm [11] in details. The original centralized algorithm consists of the following main phases: 
(i) compute the road frequency; (ii) remove infrequent roads from trajectories; (iii) cluster similar trajectories and compute the representative trajectory for each cluster. Moreover, the centralized algorithm needs to scan the trajectory dataset twice, one for road-frequency computation and the other for trajectory clustering.

After examining the detailed anonymization steps, we propose three-rounds of map-reduce processes, which ensures that the whole dataset is scanned only once. Specifically, we parallelize the road-frequency calculation in the first round of process. Then, we examine the road frequency in parallel and remove infrequent roads. While examining the road frequency, we also build an index that group same trajectories together to prepare for the clustering. Finally, we anonymize the different subtrees of the trajectory index in parallel.

In what follows, we elaborate the detailed algorithms in the map-reduce programming style, which consists of two major functions, "map" and "reduce." To put it simple, the map function is in charge of "divide," while the reduce function is in charge of "conquer."

\section{- Round 1: Trajectory Assignment}

In this first round, the map function takes raw trajectory data as input, whereby trajectories are still represented as coordinates. The number of reducers corresponds to the number of regions we obtained during the map partition phase. The map function will first map the trajectories to a region in the road network map. Specifically, the map function assigns an initial score of 0 to each region on the map for the current trajectory, and increases the score of the region by 1 when a road segment in the trajectory is found belonging to that region. Recall that each road has already been marked with the region ID during the map partitioning.

When the entire trajectory has been analyzed, the mapper finds the region with the highest score for the current trajectory. If the region contains more than $\alpha \%$ of the trajectory, then the trajectory is considered to belong to that region. Otherwise, we may split the trajectory across two regions that contain the majority of the trajectory. While the value of $\alpha \%$ has no affect on run times in our experiments, it does affect the data utility rate. The greater the value of $\alpha$ the more likely we are to place the trajectory in the correct area with the most similar trajectories. A small $\alpha$ could lead to the same problems we have when naively partitioning the map. In the case where one area does not contain more than $\alpha \%$ of the trajectory, we propose two methods: (i) splitting the trajectory as mentioned above; (ii) consider the entire trajectory as part of the region that contained the greater portion of the trajectory.

When the correct region has been identified, the mapper then converts the coordinates in a trajectory to a list of road IDs. The conversion to the road IDs makes it easier for trajectory comparison and frequency computation and reduces the intermediate data size. Finally, the map function outputs two types of key-value pairs. One is $\left\langle\left(A_{i}, \operatorname{Tr} j\left(R_{1}, \ldots R_{k}\right)\right), 1\right\rangle$, where $A_{i}$ is the region of the trajectory $\operatorname{Tr} j$ represented by road IDs $\left(R_{1}, \ldots R_{k}\right)$. Also, for each road examined, the map function outputs a key value pair $\left\langle\left(A_{i}, R_{j}\right), 1\right\rangle$, where $A_{i}$ is the region ID of the road $R_{j}$. In each mapper, we further utilize a combiner to sum up the frequency of each trajectory and each road to reduce the amount of intermediate results to be sorted and shuffled among the follow-up reducers.

The reduce function then computes the sum (or frequency) of each trajectory and each road. Note that each reducer handles one region of the map, thereby keeping similar trajectories together. The road information is saved into a hash table while the trajectories are output to an intermediate file. The final output of this round is the result of a key-value conversion, where trajectory information has been moved from the key section to the value section, so that we obtain trajectories for each map partition: $<A i,\left(\operatorname{Trj}_{i}\right.$, count $)>$.

To better understand our approach, we use the following running example throughout the article. Suppose that we have the map and trajectory data for a city. For easy illustration, we partition the map into four regions denoted as $A 1, A 2, A 3$, and $A 4$, respectively. Let the anonymization 
parameter $k=3$ and the trajectories in our data set be $\operatorname{Trj}_{1}, \operatorname{Trj}_{2}, \ldots, T r j_{n}$, whereby $x_{i j}$ and $y_{i j}$ denote the $x$ and $y$ coordinates for the $j$ th node in the $i$ th trajectory:

$\operatorname{Trj}_{1}=\left\{\left(x_{11}, y_{11}\right),\left(x_{12}, y_{12}\right), \ldots,\left(x_{1 i}, y_{1 i}\right)\right\}$

$\operatorname{Trj}_{\mathrm{n}}=\left\{\left(x_{n 1}, y_{n 1}\right),\left(x_{n 2}, y_{n 2}\right), \ldots,\left(x_{n j}, y_{n j}\right)\right\}$.

These trajectories are sent to mappers, and the mappers would map each trajectory to a region and convert the coordinates to road ID's. Assuming that these trajectories are mapped to region $A 1$, the output from this first round of map-reduce contains two types of key-value pairs, the trajectories and their assigned areas as well as each road segment and their assigned area, as follows:

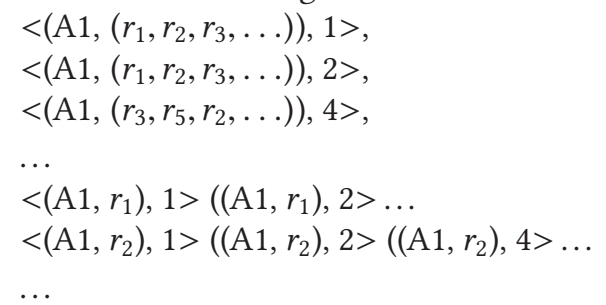

The reducer computes the overall frequency of trajectories and roads and output the following: $<\left(\mathrm{A} 1, r_{1}\right), 4><\left(\mathrm{A} 1, r_{2}\right), 4><\left(\mathrm{A} 1, r_{3}\right), 4><\left(\mathrm{A} 1, r_{4}\right), 3>\ldots$

$\left.<\mathrm{A} 1,\left(\left(r_{1}, r_{2}, r_{3}, \ldots\right)\right), 3\right)>$,

$\left.<\mathrm{A} 1,\left(\left(r_{3}, r_{5}, r_{2}, \ldots\right)\right), 4\right)>$,

$\ldots$

\section{- Round 2: Pre-Classification}

In this round, the mappers will first process all the key-value pairs containing road frequency. We leverage the sorting in the shuffle-sort phase to ensure road-frequency information arrives before trajectory frequency. For each road, the mapper checks its frequency. If the frequency is lower than the anonymization threshold $k$, then the mapper records this infrequent road ID in a hash table.

Although it is now possible to execute the centralized anonymization algorithm directly on the above dataset in the reducers, we propose another round of map-reduce to further parallelize the anonymization process. Specifically, the centralized anonymization algorithm requires to construct a C-Tree [11] to group trajectories based on similarity before final anonymization. The original C-Tree construction algorithm considers all the trajectories in a sequential manner. Instead, we propose a parallel algorithm to build the C-Tree in a distributed fashion, which also enables the subsequent anonymization to be executed in sub-trees in parallel.

To construct the C-Tree in parallel, the reducers create a list of slots whereby each slot corresponds to a branch of the original large C-Tree. The number of branches chosen depends on the number of available reducers and the size of the data, meaning that in the final round we will utilize more reducers and leverage map-reduce's batch processing at the reduce phase to process all branches of each C-Tree faster. Similar to the map partitioning in the beginning, we want to make sure trajectories most similar to one another are placed into the same branch just as they would in the original C-Tree. We also observe that the density of the data plays a role in the partitioning as well. Dense data, that is trajectories all close together, can be broken into more branches than sparse data. An example of branching is shown in Figure 4.

For each trajectory, if a branch exists such that the trajectory is at least $\gamma$ similar to that branch, then the trajectory is assigned to the same branch. $\gamma$ is a system parameter that controls how similar the trajectories in each branch are to one another. A $\gamma$ value of $100 \%$ means the trajectories are identical. Otherwise, if there are unassigned branches, then the trajectory is assigned to a new 


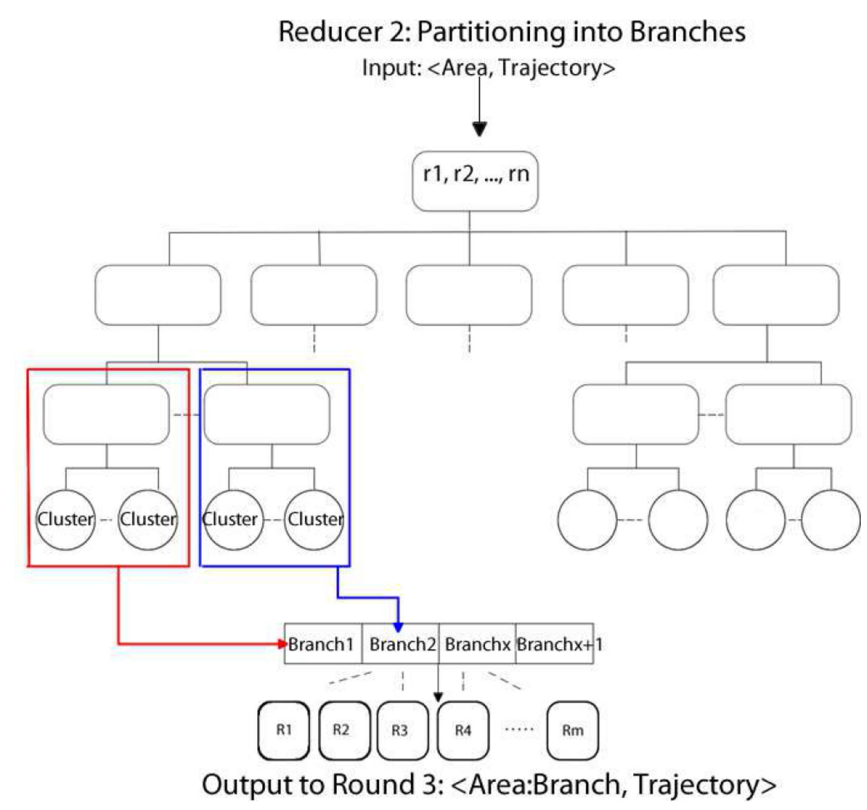

Fig. 4. In the second reducer phase, instead of using one large c-Tree, we break off similar branches into smaller chunks to benefit from map-reduce's batch processing in the reducers.

branch. Finally, if there is no more room for more branches and the trajectory is not very similar to the given branches, then it is assigned to the most similar branch. This means that the most similar trajectories will be assigned to the same branch, minimizing errors as defined in Section 3.3. The output is represented as $\left\langle\right.$ Part.id, (Traj $j_{i}$, support) $)$, where Part.id is the partitioning id consisting of the original region of the map concatenated with the branch index the trajectory was assigned to. The design of the key ensures that each area is broken up into multiple branches and processed in chunks in the final stage, rather than all at the same time, while still making sure to keep the most similar trajectories in the same chunk.

Referring to our running example, using the road frequencies in the hash table for the correct area, we remove all infrequent roads from the trajectories and output the new trajectories with only the frequent roads. As a result, our trajectories have been converted to the following:

$$
\begin{gathered}
\operatorname{Trj}_{1}=r_{1}, r_{2}, r_{3}, \\
\operatorname{Trj}_{2}=r_{1}, r_{2}, r_{3}, \\
\operatorname{Trj}_{3}=r_{1}, r_{2}, r_{3}, \\
\operatorname{Trj}_{4}=r_{1}, r_{2}, r_{3}, r_{4}, \\
\operatorname{Trj}_{5}=r_{6}, r_{7}, \\
\operatorname{Trj}_{6}=r_{6}, r_{7}, \\
\operatorname{Trj}_{7}=r_{2}, r_{3}, r_{6}, r_{7} .
\end{gathered}
$$

If we remember that we set $k=3$, then in $T r j_{4}, r_{4}$ would be removed for being infrequent. The trajectories are sent to the second reducers where trajectories 1, 2, 3, and 4 are partitioned to a branch, and the remaining would be grouped in another branch based on their similarity.

\section{- Round 3: Melt}

This final round of our algorithm conducts trajectory anonymization in parallel. The algorithm is outlined in Algorithm 2. The mappers here are just identity mappers, which directly pass the datasets obtained from the previous step to the reducers without additional process. Each reducer 


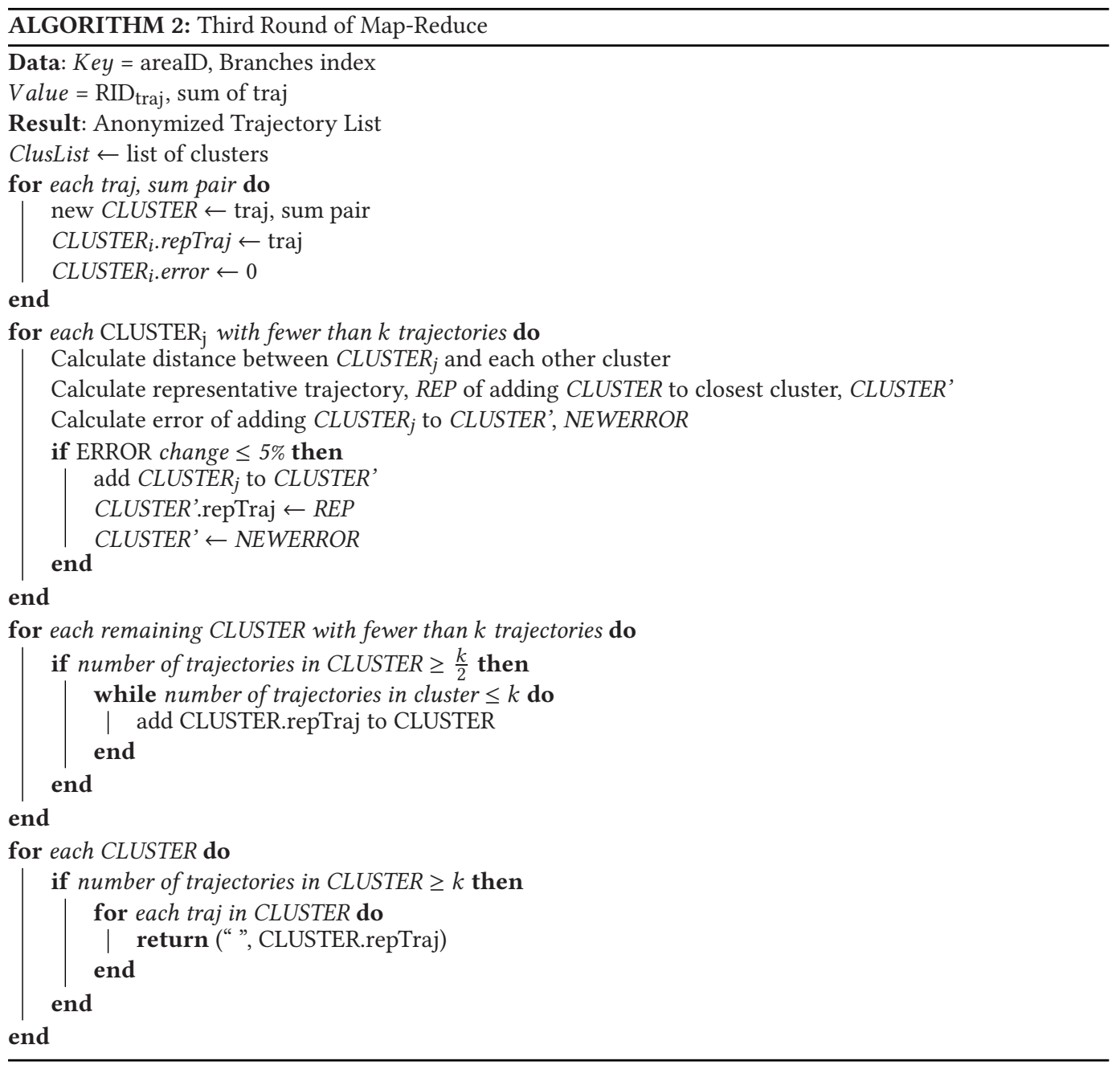

will receive the dataset that corresponds to a single branch of the C-Tree, and construct the C-Tree as defined in Reference [11]. nlike a two round algorithm where an entire area would be anonymized at once in each reducer, processing branches instead of entire areas significantly increases efficiency by taking advantage of map-reduce's's batch processing and load balancing. More specifically, for each trajectory, if the total support is greater than $k$, it forms a cluster and is added to the tree. If the trajectory has a support lower than $k$, then it will traverse the tree and find the most similar cluster and attempt to merge with that cluster. Note that this is significantly more efficient than the centralized approaches, which need to compare trajectories in the entire dataset, not a small subset such as in our approach. When merging, a representative trajectory is calculated for the cluster and error is calculated.

When we attempt to merge trajectories with low frequency into a cluster, we calculate the representative trajectory of the cluster as though the trajectories were added and if the error is increased by less than $\beta \%$, the trajectories are added. The error threshold is an adjustable system parameter, which can also be set by the user depending on the amount of error that is acceptable to them. If no clusters are suitable for merging, then a new cluster is created for the trajectory and added to 
the tree. By doing this, we are able to possibly merge multiple trajectories with low support together to meet the $k$ threshold, should they be suitably similar. When all possible merges are done, representative trajectories of any clusters with at least $k$ support will be output. Any trajectories remaining with less than $\frac{k}{2}$ support will be removed from the data set. Any remaining trajectories with more than $\frac{k}{2}$ support, but less than $k$ will have false trajectories added, increasing the support to $k$. This is done to increase data utility while keeping error low and preserving privacy. The final anonymization output contains road IDs and road frequency in the representative trajectories of all the clusters. Our anonymization result guarantees strict $k$-anonymity.

Finishing up our running example, in the last round of reducers, trajectories 1, 2, and 3 would be put into a cluster, since their frequency is greater than $k$ and the representative trajectory would be calculated. $\operatorname{Tr}_{4}$ would also be merged into this cluster, since after the infrequent road was removed and no additional error is added, it now matches this cluster. In another reducer, we cluster trajectories 5 and 6; however, if we try to merge $\operatorname{Tr}_{7}$ with trajectories 5 and 6 , then the error is too great and is placed in its own cluster. Since the frequency for $\operatorname{Tr} j_{7}$ is less than $\frac{k}{2}$, it is removed entirely. The frequency for the cluster containing trajectories 5 and 6 however, is greater than $\frac{k}{2}$. So we simply add a dummy trajectory to it to bring the frequency up to $k$. The final output of the anonymized set would be

$$
\begin{array}{llll}
r_{1}, r_{2}, r_{3} & r_{1}, r_{2}, r_{3} & r_{6}, r_{7} & r_{6}, r_{7} \\
r_{1}, r_{2}, r_{3} & r_{1}, r_{2}, r_{3} & r_{6}, r_{7} &
\end{array}
$$

The trajectories are now $k$-anonymized. The data still maintains utility and individual users cannot be identified.

\subsection{Privacy Preservation with Varying $k$}

So far in this work, the privacy of users depends on a globally fixed $k$ value chosen before trajectories are anonymized. However, for some users, their privacy requirements may vary compared to other users. This can be observed in many social media environments. One user may share everything to the public, while others choose not to use social media at all due to privacy concerns. While these two examples represent both extremes, there are users all across the spectrum between them. Therefore, we propose to extend our approach to accommodate users with different privacy requirements and allow each individual user to set his/her own value of $k$. As of now, this is the first work to allow user control for their privacy while maintaining the high efficiency and accuracy of the final results.

To achieve variable $k$ during the anonymization, we propose the following modifications to our parallel algorithm. The first change is to maintain the personal $k$ value for each trajectory throughout all the three rounds of parallel processes. Note that although it seems that keeping the personal $k$ value attached to the user may be more space efficient, it is impossible to distinguish users, since no identifiers or even quasi-identifiers are stored in the system.

The second change occurs in the third round of anonymization, particularly during the output of representative trajectories. When deciding whether or not to output a cluster, we will check to make sure each individual $k$ requirement is met. The naive solution is to compare each personal $k$ with its cluster support. If the cluster support is lower than one of the users' $k$, meaning that the user's privacy is not met, then the cluster will be removed. However, this naive solution can result in removing a large number of clusters simply due to some users who have a higher $k$ value than their cluster members. To prevent loss on the data utility rate, we propose the following algorithm that attempts two options. Instead of directly removing the whole cluster when its support is lower than some users' $k$ values, our first option is to try to increase the cluster support and recalculate the error. If the introduced error is within the desired threshold, then the cluster will be preserved. 
If the first option does not work, then we try the second option, which is to remove the trajectory with particularly high $k$ from the cluster and recalculate the representative trajectory for that cluster. This additional step provides users with more controls on their privacy while introducing very little time overhead to the performance as later shown in Section 6.

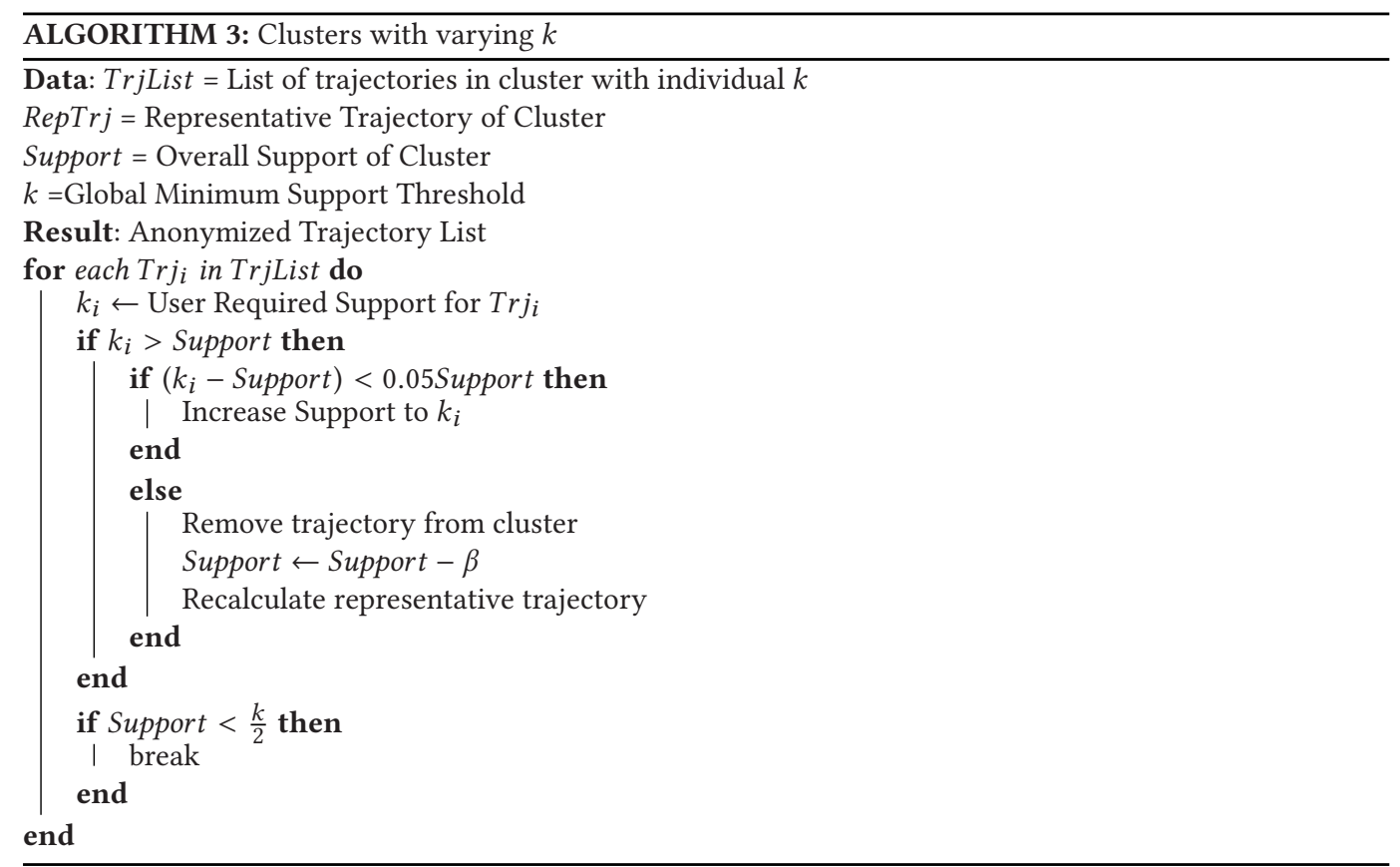

\section{SYSTEM ANALYSIS}

\subsection{Privacy and Utility Analysis}

In general, the more different the anonymized dataset is from the original dataset, the higher level of privacy will be preserved, since it makes it harder for attackers to infer the original information. However, too much divergence from the original dataset causes information loss and makes it hard to conduct any meaningful data analysis. Therefore, it is important to achieve the balance between the privacy preservation and data utility preservation during the trajectory anonymization. In what follows, we show that our approach closely align with three important guidelines about trade-offs between privacy and utility as stated in Reference [29].

The first guideline is that knowledge gained on a small population or individual has the largest impact on privacy, while knowledge gained overall on a population increases utility. This means that privacy must be considered on an individual basis rather than on an entire population, while information gained on an individual has little utility compared to an entire population. In our work, we consider each individual's privacy to be unique and important, therefore, our work has a large impact on user privacy. Similarly, we are looking for large groups of trajectories to study and gain knowledge on patterns, therefore we also have a large utility gain.

The second guideline is that privacy should be measured at its worst case scenario for reasons stated above while utility should be measured at its best case. This means that the unacceptable privacy loss of even a single individual should be considered the privacy loss for the entire population in the data while utility should be looked at as how much information is ultimately gained. In 
our research, we observe that no individual's data is published if it is below the threshold $k$. Thus, it is appropriate to measure privacy loss in terms of $k$, since we do not have any quasi-identifiers in our datasets. Additionally, since we allow users to choose their own level of privacy and we consider it as a strict constraint, we can say that we focus strongly on individual privacy. Similarly, we always look at the highest and average precision and recall to determine data utility. This means we look at the best and average utility of the final data sets and therefore measure at its best case.

The third guideline is that privacy is to be measured against another anonymized dataset to determine if privacy is maintained, improved, or lost while utility should be measured against the original dataset to determine how much of the original data is maintained. In our tests, we compare privacy to the anonymized data produced by the centralized approach in Reference [11]. This is to make sure that through our divide-and-conquer technique, we are maintaining the same privacy standards and not unintentionally relaxing our privacy requirement. We measure our utility by calculating the number of trajectories retained from the original data set and how similar those trajectories are to determine how much utility the anonymized dataset has.

In our work, we calculate utility loss by calculating the precision and recall of the results from our approach compared to the centralized approach and the original data set to determine: (i) How much of the original dataset we retained and (ii) How similar are our results compared to the centralized and original data. This allows us to see what percentage of the data we kept after anonymization and how much error we introduced by our approach.

Privacy loss, however, is more challenging to calculate. While we can give an arbitrary $k$ value that sounds secure enough, we choose to use the measurement included in Reference [29] Ploss = $\frac{\max }{t}$ Ploss $(t)$. This calculation matches the second guideline, that privacy should be measured at its worst case and considered for the entire population. Since there is not any user identifier or quasiidentifier in our dataset, there is no need to calculate privacy loss for each individual's attribute. Instead, we look at privacy on each hop in a trajectory for a user and calculate it as follows:

$$
\text { Ploss }=\sum_{i=1}^{h}\left(P\left(n_{i}\right) \cdot 2 P\left(\sum_{j=1}^{m}\left(t_{i}\right)\right)\right),
$$

where $P\left(n_{i}\right)$ is the probability that an adversary can identify an individual user from published users on a road segment. $2 P\left(\sum_{j=1}^{m}\left(t_{i}\right)\right)$ addresses the inference-route problem in that it is the probability that both a user chose to take an infrequent road and that the adversary was able to correctly choose the same road in identifying the user. Since infrequent roads are not published, this is considered very random for both the user and the adversary. There are possibly several infrequent roads a user can take, therefore it is a summation of all those possible paths. We can make it a little more simple by remembering that the minimum number of people on a published road segment is $k$ and the worst case being that no unknown, infrequent paths were taken. Therefore, we can say that for $i$ hops in a published trajectory, privacy loss can be calculated as

$$
\text { Ploss }=\sum_{i=1}^{h}(P(k)) .
$$

Since we do not use any identifiers or quasi-identifiers in our dataset and we remove infrequent roads to protect against the inference-route problem, we can say that $k$ is a sufficient measure of privacy loss, since we follow all of the privacy guidelines in Reference [29].

\subsection{Complexity Analysis}

In this section, we will look at each round of our algorithm and determine the worst and average case scenario. Map partitioning is not analyzed, as performing this task requires only the list of 
road intersections and a small sample set of the data. The size of this data is trivial compared to the trajectory dataset. As mentioned earlier, we only scan the entire dataset once. Therefore, the processing time is mainly dominated by the second-round produce of our algorithm.

5.2.1 Round 1 Analysis. In the first round, we examine the time taken to assign trajectories to map partitions and output each trajectory and the roads in those trajectories as well as their assigned partitions. Based on this, the time for round 1 is $\frac{c n+n}{x}$, where $c$ is a constant representing the average number of nodes in a trajectory, $n$ is the number of trajectories, and $x$ is the number of map partitions.

5.2.2 Round 2 Analysis. The data to reach the second round will be the roads and their assigned map partitions. The first portion of round 2 is to identify infrequent roads. The processing time for this can be represented as $\frac{m}{i}$ where $m$ is the number of roads and $i$ is the number of mappers. According to the number of occurrences of roads, we remove the infrequent roads from the trajectories, which takes the time $\frac{c n}{i}$.

Once infrequent roads are removed, we perform the pre-clustering algorithm. We represent the number of chunks we are breaking the data into as $j$ and the value we define for $k$-Anonymity as $k$. In the worst case scenario, there will be only one reducer and each kind of the trajectory has no more than $k$ similar trajectories. This will result in the maximum number of chunks of data. The amount of calculation can be represented as $\frac{n * \frac{n}{k}}{j} . j$ is an arbitrary value set by the user and is calculated based on data size, therefore we can say the worst case scenario is $\frac{n^{2}}{k}$. Finally, we consider the $\mathrm{I} / \mathrm{O}$ cost as $\frac{n}{x}$.

5.2.3 Round 3 Analysis. In round three, each of the data chunks is put into C-Trees, and the representative trajectory of clusters meeting our $k$ requirement are the output. For each trajectory, the algorithm finds the correct cluster in the C-Tree. The time to find these clusters is $n \log (\mathrm{cn})$, which is split over $x$ reducers. However, in the previous round, we split the data going to the reducers into $j$ chunks, therefore the time is $\frac{x n \log (c n)}{j}$. For each of these clusters, we calculate a representative trajectory: $\frac{c n}{x}$, and finally output the representative trajectories of the clusters that meet the $k$ requirement: $\frac{n}{k}$.

5.2.4 Overall Complexity. The final result for the worst case scenario is: $O\left(n^{2}+k \log (c n)\right)$. However, for the second round, the chance that every trajectory will need to be compared to every other trajectory is very small. Additionally, the chance that the data will be clustered into sizes of exactly $k$ are even smaller. The average case would more realistically mean the correct chunk of data to assign a trajectory to can be found in $\frac{n \log (j)}{x}$ time making the average run time $\theta(n+n \log (c n))$.

\section{PERFORMANCE STUDY}

In this section, we first present the experimental settings and then report the experimental results.

\subsection{Experimental Settings}

All the tests were conducted on a cluster of four physical machines, each being Dell PowerEdge R730, Intel Xeon CPU E5-2650 v3 processors at $2.30 \mathrm{GHz}, 2$ sockets, 14 cores per socket. The machines are managed using VMWare and divided up into eight virtual machines, each running CentOS 64bit and having 14 CPU's and 60 GB RAM. We used Apache Hadoop 2.3.0 and Spark 2.3.0.

Our experiments were conducted on both real and synthetic datasets. The real datasets [33] contain taxi data from drivers in New York City over a period of three years, all taken from the same three hour time frame to consider the potential privacy issues stemming from temporal information. The real datasets are represented in Table 2 and contain the data file sizes as well as 
Table 1. The Number of Trajectories and the Corresponding File Size

\begin{tabular}{l|c|c|c|c|c|c|c|c}
\hline Number of Trajectories & $5 \mathrm{~K}$ & $50 \mathrm{k}$ & $500 \mathrm{k}$ & $2 \mathrm{M}$ & $5 \mathrm{M}$ & $50 \mathrm{M}$ & $250 \mathrm{M}$ & $500 \mathrm{M}$ \\
\hline \hline Data Size & $3 \mathrm{M}$ & $14 \mathrm{M}$ & $141 \mathrm{M}$ & $540 \mathrm{M}$ & $1.4 \mathrm{G}$ & $14 \mathrm{G}$ & $55 \mathrm{G}$ & $103 \mathrm{G}$ \\
\hline
\end{tabular}

Table 2. Real Trajectory Datasets Tested for Efficiency

\begin{tabular}{l|c|c|c|c}
\hline Dataset Name & RS & RS50 & RS100 & RS200 \\
\hline \hline Number of Trajectories & $17 \mathrm{~K}$ & $175 \mathrm{M}$ & $420 \mathrm{M}$ & $862 \mathrm{M}$ \\
\hline Data Size & $137 \mathrm{~K}$ & $50 \mathrm{~GB}$ & $100 \mathrm{~GB}$ & $200 \mathrm{~GB}$ \\
\hline
\end{tabular}

the number of trajectories. Note that one smaller dataset was taken in order for the centralized and single round approaches to be able to process it and compare accuracy. In addition to the real datasets, we also generate several large synthetic datasets over real road maps so that we can show our approach is consistent over several sets of data and locations. The synthetic datasets contain trajectories with similar average length in the real datasets, which is about 15 to 20 nodes. The trajectory node distribution is random to avoid any bias. The synthetic dataset size goes up to 100 GB, which is sufficient to represent commuter traffic in a large city such as Los Angeles or New York City over a three month period [4]. Throughout our experiments, we refer to our datasets by the number of trajectories in the input data file. Table 1 presents the statistic summary of the synthetic datasets while Table 2 presents the real datasets.

In our experiments, we compare the performance of the following algorithms in terms of efficiency and effectiveness.

- Centralized: The centralized anonymization approach proposed in Reference [11].

- Single-round MapReduce: This approach executes the original centralized anonymization algorithm directly on each sub-dataset after the map partitioning.

- Stripe: The map is partitioned into even sized stripes and then trajectories are anonymized using our proposed parallel approach.

- Grid: The map is partitioned into even sized squares and then trajectories are anonymized using our proposed parallel approach.

- MBR: The roads in the map are partitioned using minimum bounding rectangles (MBR) such as those in the R-trees. Specifically, we divide the roads into equal-sized groups based on their distance and enclose them in minimum bounding rectangles to form partitions.

- MELT-Hadoop: The full version of our proposed MELT algorithm in MapReduce.

- MELT-Spark: The full version of our proposed MELT algorithm in Spark.

For the map partitioning, we compare our solution with the naive partitioning methods, as described in Section 3. To evaluate the anonymization accuracy, we compare our results with the centralized approach proposed by Reference [11], as well as a single round map-reduce algorithm and naive partitioning methods. We test the effects of changing the $k$-threshold, the number of map partitions, dataset size, and multiple map topologies. Finally, we test the effects of having user defined $k$ on the data utility and processing time. Effectiveness is evaluated based on the precision and recall defined in Section 3. Efficiency is evaluated using CPU time.

\subsection{Effect of Map Partitioning on Anonymization Accuracy}

In the first round of experiments, we aim to study how map partitioning will affect the anonymization accuracy. We compare our dynamic partitioning approach to the naive partitioning methods 


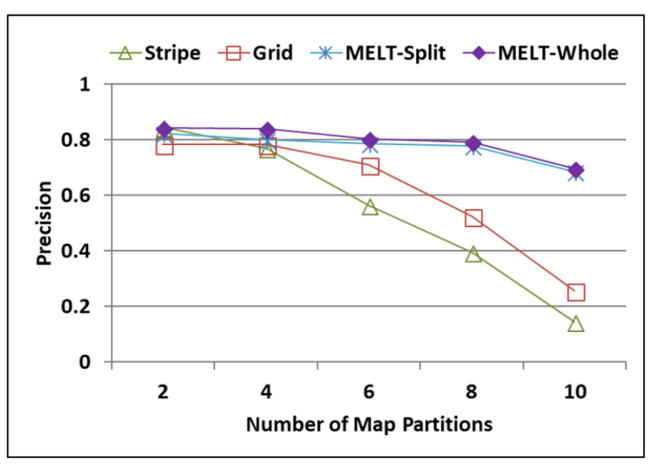

(a) Precision

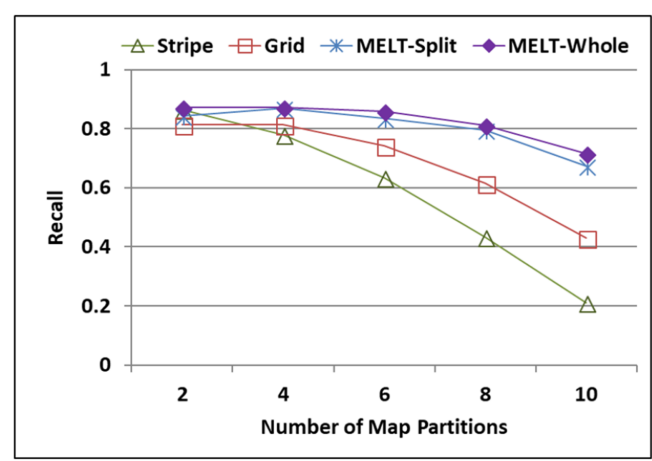

(b) Recall

Fig. 5. Anonymization accuracy when dividing the map into different number of partitions.

introduced in Section 3 and the approach using the simple minimum bounding rectangles (MBRs). More specifically, our approach has two versions: MELT-whole and MELT-split. In the MELTwhole approach, we always keep the trajectories as a whole even if it crosses partitions, and assign the trajectory to the partition in which the majority of the trajectory lies. The MELT-split approach, instead, splits the trajectory if it crosses partitions, and assign each part of the trajectory to its respective partition. Figure 5 shows the anonymization accuracy of all the approaches in terms of precision and recall. Recall that both precision and recall need to be calculated against the original anonymization results produced by the centralized approach. Since the centralized approach cannot handle very large datasets, in this experiment, we adopt the largest dataset that the centralized approach can handle, which contains $500 \mathrm{~K}$ trajectories and set the anonymization parameter $k$ to $0.5 \%$ of the number of trajectories.

As show in the figure, both versions of our proposed MELT yield significantly higher accuracy results compared to other approaches especially when the number of partitions is large. This conforms with our analysis that equally partitioned maps do not match the trajectory distributions as well as our proposed dynamic partitioning. Also, since our approach performs even better for the larger number of partitions, it indicates the advantages of our approach in large-scale datasets, which typically needs more partitions to handle. We also observe that the MELT-whole achieves slightly better accuracy than MELT-split, since MELT-whole would be able to keep more trajectories intact. In addition, it is expected that the more partitions, the lower the anonymization accuracy. This is because when the number of partitions is large, the area of each partition becomes small and hence more trajectories may cross partitions resulting in more difficulties in identifying similar trajectories. However, more partitions could help reduce the overall processing time for large-scale datasets, since each partition handles a smaller amount of data. Therefore, we carefully choose the number of partitions based on the dataset size in the following experiments to balance the anonymization accuracy and the anonymization time.

\subsection{Effect of the Anonymization Parameter $k$ on Data Utility}

As we already know that the proposed MELT has better anonymization accuracy than other approaches, we now take a further look only at the anonymization results of the MELT in terms of data utility. Note that both MELT-Hadoop and MELT-Spark versions have the same data utility rate, since they implement the exactly same algorithms.

Specifically, we investigate the effect of the anonymization parameter $k$ on data utility in terms of the number of trajectories retrained in the anonymization result. Since $k$ may have more impact 
Table 3. Number of Trajectories in the Anonymization Results

\begin{tabular}{c|ccc}
\hline Number of trajectories in the dataset & $5 \mathrm{~K}$ & $50 \mathrm{~K}$ & $500 \mathrm{~K}$ \\
\hline$k=0.1 \%$ of data & 4,255 & 35,809 & 285,995 \\
$k=0.5 \%$ of data & 3,228 & 30,910 & 279,500 \\
$k=1 \%$ of data & 878 & 18,626 & 132,602 \\
\hline
\end{tabular}

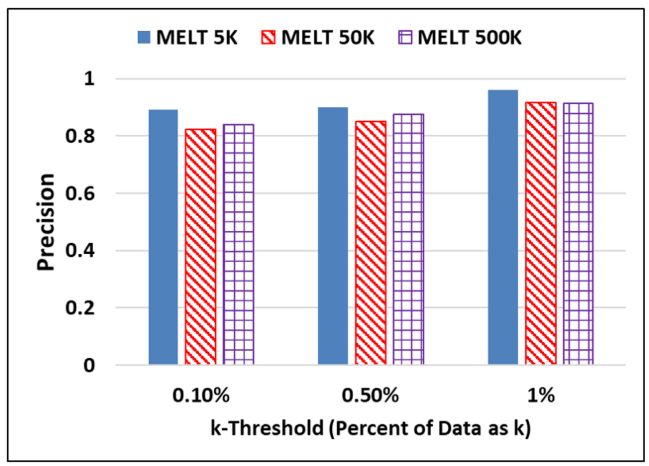

(a) Precision

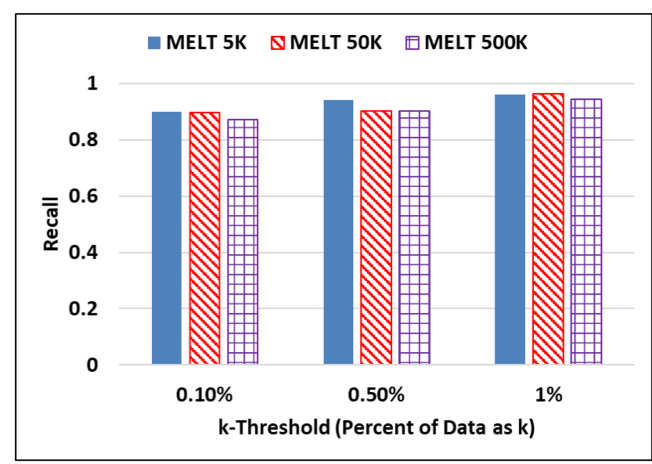

(b) Recall

Fig. 6. Anonymization accuracy when varying $k$.

on smaller datasets, we tested three datasets ranging from as small as $5 \mathrm{~K}$ to $500 \mathrm{~K}$ trajectories. For each dataset, we vary $k$ from $0.1 \%$ to $1 \%$ of the number of trajectories.

Table 3 shows the number of trajectories left after the anonymization. As expected, the smaller dataset with $5 \mathrm{~K}$ trajectories ends up with just $20 \%$ of the original trajectories after the anonymization when $k=1 \%$ of the number of total trajectories (i.e., $k=50$ ). The larger the datasets, the more percentage of trajectories are kept after the anonymization. This is because in the larger dataset, the chances of finding similar trajectories is higher and hence more trajectories can be anonymized. In addition, we also observe that there is a significant reduce in the number of anonymized trajectories when $k$ increases from $0.5 \%$ to $1 \%$, which suggests that the anonymization parameter $k$ should not be set to be too large. The larger the $k$ value, the lower the probabilities will be to obtain similar trajectories in the group larger than $k$.

Next, we are also interested in examining the effect of $k$ on the precision and recall. As shown in Figure 6, we can see that there is little difference in anonymization accuracy when varying the $k$ values. The possible reason is that the precision and recall are computed by comparing the anonymized results from the centralized approach and our parallel approach. Either centralized approach or parallelized approach will yield much fewer trajectories with the increase of $k$. In other words, the precision and recall do not represent the data utility loss.

\subsection{Scalability Test}

After obtaining the insights regarding the map partitions and the parameter $k$, we now proceed to evaluate the scalability of our proposed MELT, which is indeed the major goal of our design. We first tested the centralized annonymization approach to see its scalability limit. As shown in Table 4, the centralized approach crashes when the dataset contains more than $2 \mathrm{M}$ trajectories.

Since the centralized approach is impossible to complete the anonymization as shown in Table 4, we only plot the processing time of the parallel approaches in the following figures for clear 
Table 4. The Anonymization Time Using the Centralized Approach

\begin{tabular}{c|ccccc}
\hline Number of trajectories in the dataset & $5 \mathrm{~K}$ & $50 \mathrm{~K}$ & $500 \mathrm{~K}$ & $2 \mathrm{M}$ & RS (17K trajectories) \\
\hline File size & $3 \mathrm{M}$ & $14 \mathrm{M}$ & $141 \mathrm{M}$ & $540 \mathrm{M}$ & $137 \mathrm{M}$ \\
\hline Processing time (minutes) & 0.9752 & 22.6 & 1157.72 & NA & 1441.49 \\
\hline
\end{tabular}

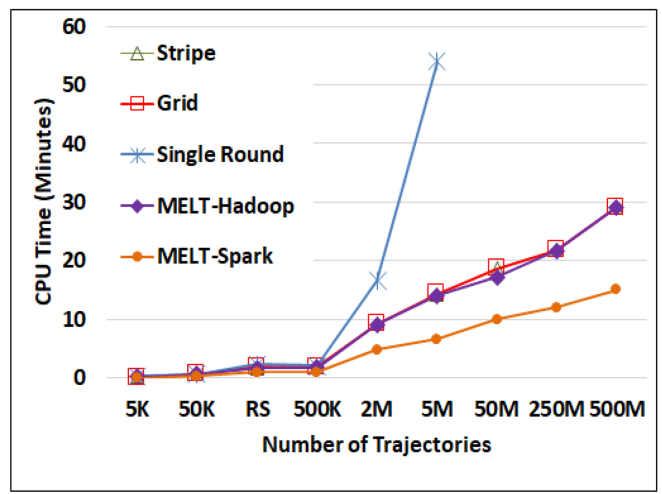

(a) Synthetic Datasets

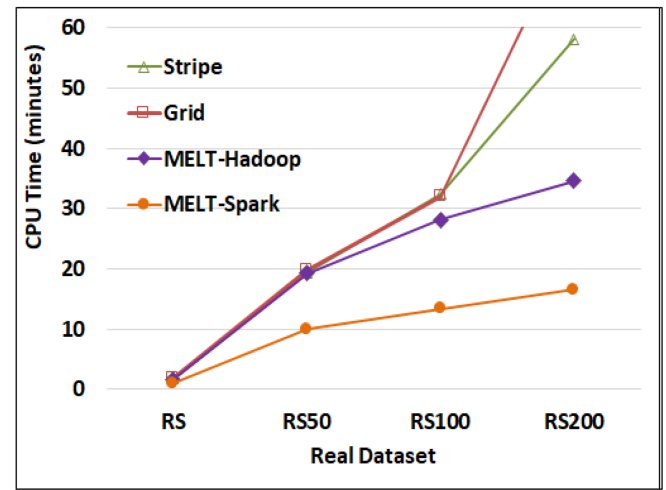

(b) Real Datasets

Fig. 7. Effect of data size on anonymization time.

comparison. In the experiments, the number of partitions is set to 8 and the value of $k$ is set to $0.5 \%$ of the number of trajectories. As shown in Figure 7, the full version of the MELT-Hadoop is similarly fast as the MELT version with simple map-partitioning methods, i.e., stripe and grid. This indicates that our dynamic map partitioning does not introduce much computational overhead. Moreover, we also observe that the single-round MapReduce approach does not scale well and crashes when the number of trajectories reach $5 \mathrm{M}$. This proves the need of having multi-round of divide-and-conquer as in the full version of the MELT. Also, it is not surprising to see that the Spark version of MELT is about twice faster than the Hadoop version. The performance gain is attributed to the in-memory access by Spark.

In addition to the synthetic datasets, we also compare the same approaches using the real datasets in Table 2. Based on our results in Figure 7(b), our approach of MELT-Hadoop and MAPSpark show much better scalability with the growing data size, with MELT-SPARK showing more than $120 \%$ improvement in CPU time compared to MELT-Hadoop.

Further, we also compare the anonymization accuracy of all the parallel approaches. Since the anonymization accuracy is calculated by comparing the anonymization results with the centralized approach, Figure 8 shows the comparison results with the dataset up to $500 \mathrm{~K}$, which is the maximum size that the centralized approach can process. Observe that the full version of the MELT achieves much better precision and recall than the naive partitioning approaches when the dataset grows larger. Note that the full version of MELT here refers to both MELT-Hadoop and MELTSpark, since they produce exactly the same anonymization results. When the dataset is very small (say $5 \mathrm{~K}$ ), the anonymization accuracy is similar among all the approaches, because the number of trajectories in the anonymization results is small too and hence less difference. Note that only two partitions are used for $5 \mathrm{~K}$ dataset whereby the Grid partitioning approach is basically the Strip partitioning, and thus no result is reported for the Grid approach for the $5 \mathrm{~K}$ dataset.

Additionally, for the real dataset tests, we calculated the recall as recall is compared to the original dataset. We achieved an average of 0.81376 recall, meaning we maintained a high data utility 


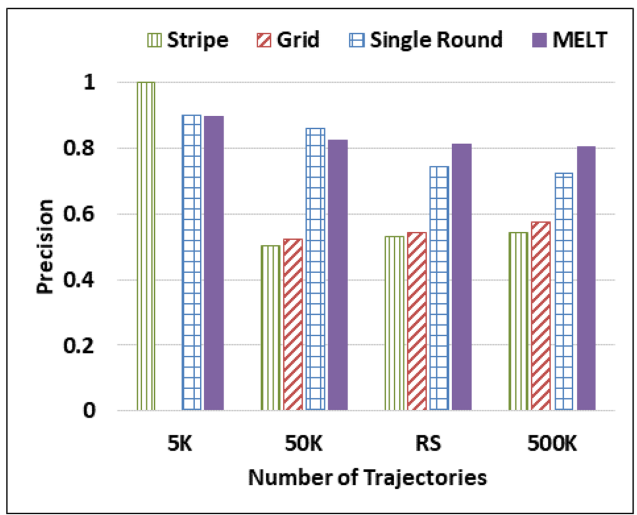

(a) Precision

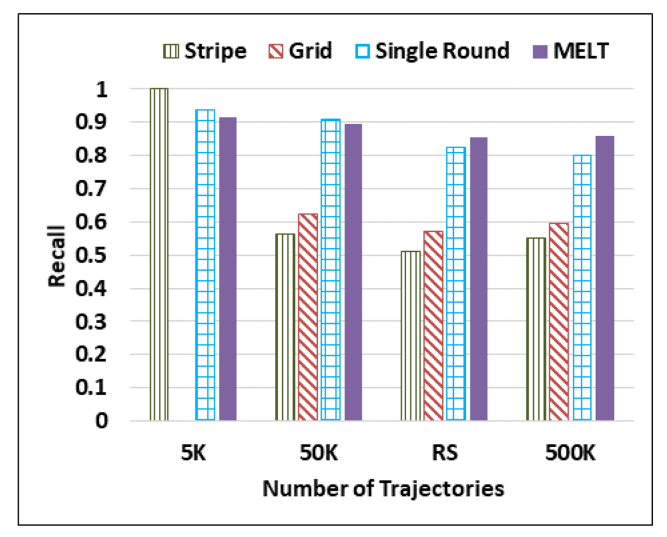

(b) Recall

Fig. 8. Effect of data size on anonymization accuracy.

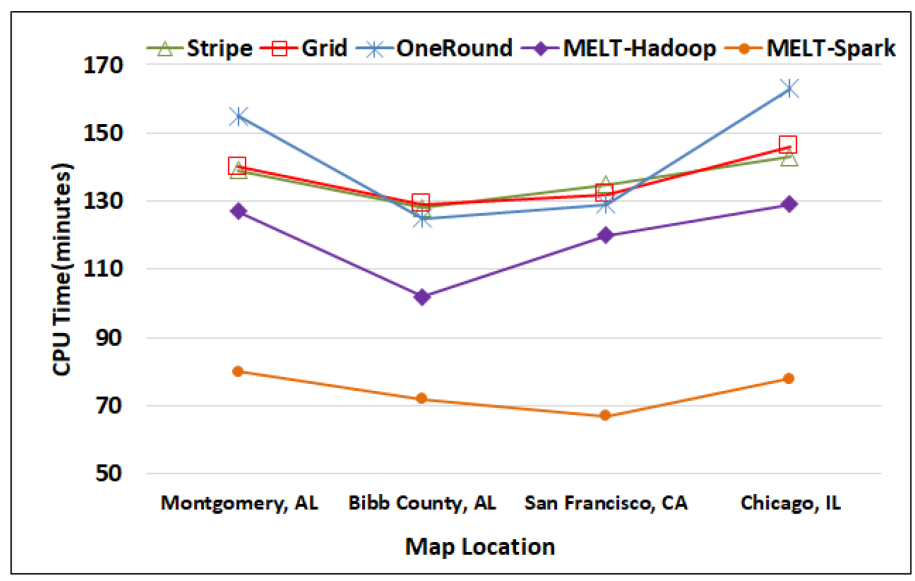

Fig. 9. Effect of map topology on anonymization time.

rate in terms of number of trajectories retained from the original dataset. Precision could not be measured as the centralized anonymization approach could not process the datasets for us to compare.

\subsection{Effect of the Map Topology on Efficiency and Accuracy}

In this experiment, we evaluate the effect of the map topology on the anonymization efficiency as well as accuracy. To measure the accuracy, we generate $500 \mathrm{~K}$ trajectories (the maximum size that the centralized approach can handle) on four different real maps representing big cities. We set the number of the partitions to 6 and $k$ to $0.5 \%$ of the number of trajectories. As we can see in Figure 9 , our proposed MELT-Spark is the fastest approach among all regardless of the map topology used. The single-round MapReduce approach is the slowest. This is because MELT, Strip, and Grid not only partition the original input dataset but also break the anonymization task into sub-tasks and hence achieve better parallelism than the single-round MapReduce.

When comparing the anonymization accuracy, MELT again achieves the highest accuracy among all as shown in Figure 10. The reason is the same as previously discussed. It is because 


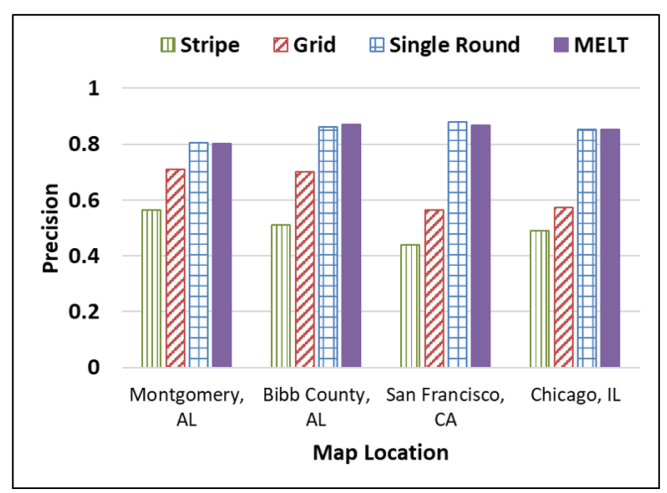

(a) Precision

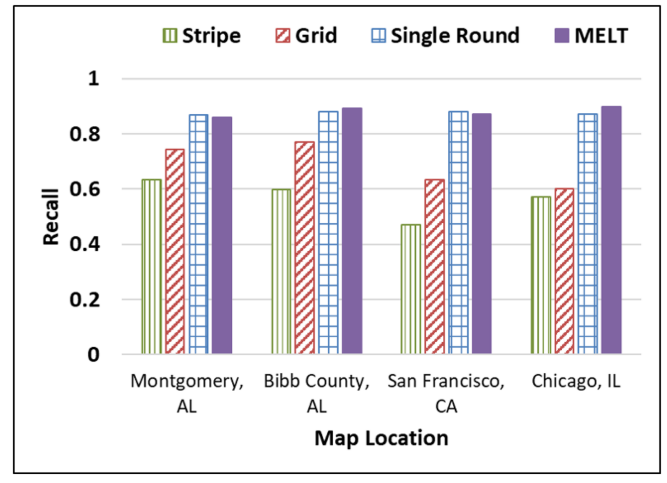

(b) Recall

Fig. 10. Precision and recall for different map topologies.

Table 5. Change of Anonymization Accuracy when Using Individual $k$ Parameters

\begin{tabular}{c|cc}
\hline Number of Trajectories & Precision Change & Recall Change \\
\hline $5 \mathrm{k}$ & -0.003 & -0.002 \\
$50 \mathrm{k}$ & -0.003 & 0.00005 \\
$500 \mathrm{k}$ & -0.009 & -0.004 \\
\hline
\end{tabular}

the equal partitioning cannot capture the real trajectory distribution. In addition, it is worth noting that the single-round MapReduce has the similar accuracy as the MELT, since they adopt the same map-partitioning approach.

\subsection{Effect of User Defined $k$ on Anonymization Accuracy}

Finally, we examine the effect of user defined $k$ on the anonymization accuracy. In the dataset of $500 \mathrm{~K}$ trajectories, for each user, we randomly generate its $k$ value ranging from 0 (no privacy protection needed) to $0.5 \%$ of the number of trajectories. Then, we compare the precision and recall between the anonymization results using the same value of $k(0.5 \%$ of the number of trajectories) and the anonymization result that used the variable $k$ for different users. Table 5 shows that the changes in precision and recall are very little after introducing customized $k$. Such good performance is because our algorithm carefully adjusts the clusters of trajectories based on individual $k s$ and maximizes the number of trajectories to be kept. The processing time after introducing the customized $k$ is also almost the same as the version with the same global $k$, and hence we did not include the figure here. The processing time is similar, because the algorithm for the customized $k$ adds only small conditions to the original version, which do not affect the overall processing time.

\section{CONCLUSION}

In this article, we propose a novel parallel algorithm called MELT to efficiently anonymize large data sets of trajectories. The proposed MELT employs three rounds of divide-and-conquer strategies that break the centralized anonymization task into sub-tasks that can be performed in parallel. The MELT also provides flexibility to individual users to define their own privacy levels. Our anonymization results on both real and synthetic datasets demonstrate that our approach successfully protects the personal data from direct knowledge or inference attacks while at the same time 
achieves high data utility rate. The extensive experimental study also shows that our method performs much better when compared with recent works in terms of computation speed, volume of data, and data utility. To further show the benefits of our work, we have also analyzed the relationship between the privacy and utility aspects of our approach. The outcome of this research can be used by cellular and other location service providers to publish anonymized trajectory data much faster, so that it can be used in a wide variety of services in timely fashion without sacrificing user privacy.

\section{REFERENCES}

[1] Osman Abul, Francesco Bonchi, and Mirco Nanni. 2008. Never Walk Alone: Uncertainty for anonymity in moving objects databases. In Proceedings of the IEEE International Conference on Data Engineering (ICDE'08).

[2] Francesco Bonchi and Hui Wendy Wang. 2011. Trajectory anonymity in publishing personal mobility data. Spec. Interest Group Knowl. Discov. Data Mining 13, 1 (2011), 30-42.

[3] Rui Chen, Benjamin C. M. Fung, Noman Mohammed, Bipin C. Desai, and Ke Wang. 2013. Privacy-preserving trajectory data publishing by local suppression. Info. Sci. 231 (2013), 83-97.

[4] Melanie Deal. 2016. Census Bureau Reports 471,000 Workers Commute into Los Angeles County, California, Each Day. Retrieved from http://www.census.gov/newsroom/press-releases/2013/cb13-r13.html.

[5] Josep Domingo-Ferrer and Rolando Trujillo-Rasua. 2012. Microaggregation- and permutation-based anonymization of movement data. Info. Sci. 208 (2012), 55-80.

[6] Ahmed Eldawy and Mohamed Mokbel. 2013. A demonstration of spatialhadoop: An efficient mapreduce framework for spatial data. Very Large Data Base 6, 12 (2013), 1230-1233.

[7] Apache Software Foundation. 2016. What is Apache Hadoop? Retrieved from http://hadoop.apache.org/.

[8] Hend Kamal Gedawy. 2009. Dynamic path planning and traffic light coordination for emergency vehicle routing. Carnegie Mellon University Thesis (2009), 1-9.

[9] Moein Ghasemzadeh, Benjamin C. M. Fung, Rui Chen, and Anjali Awasthi. 2014. Anonymizing trajectory data for passenger flow analysis. Transportation Research Part C 39 (2014), 63-79.

[10] Marco Gruteser and Dirk Grunwald. 2003. Anonymous usage of location-based services through spatial and temporal cloaking. Proceedings of the 1st International Conference on Mobile Systems Applications and Services (MobiSys'03). Vol. 3, 31-42.

[11] Sashi Gurung, Dan Lin, Wei Jiang, Ali Hurson, and Rui Zhang. 2014. Traffic information publication with privacy preservation. ACM Trans. Intell. Syst. Technol. 5, 3 (2014), 1-26. DOI : https://doi.org/10.1145/2542666

[12] Alon Y. Halevy, Michael J. Franklin, and David Maier. 2009. TRUSTER:TRajectory data processting on ClUSTERs. In Proceedings of the International Conference on Database Systems for Advanced Applications (DASFAA'09). 768-771. DOI : https://doi.org/10.1007/11733836

[13] Pin-I Han and Hsiao-Ping Tsai. 2015. SST: Privacy preserving for semantic trajectories. In Proceedings of the 16th IEEE International Conference on Mobile Data Management, Vol. 2. 80-85. DOI : https://doi.org/10.1109/MDM.2015.18

[14] Xi He, Graham Cormode, Ashwin Machanavajjhala, Cecilia M. Procopiuc, and Divesh Srivastava. 2015. DPT: Differentially private trajectory synthesis using hierarchical reference systems. Proc. Very Large Data Base Endow. 8, 11 (2015), 1154-1165. DOI : https://doi.org/2150-8097/15/07

[15] C. S. Jensen, D. Lin, and B. C. Ooi. 2007. Continuous clustering of moving objects. IEEE Trans. Knowl. Data Eng. 19, 9 (2007), 1161-1174.

[16] Xun Li, Wenwen Li, Luc Anselin, Sergio Rey, and Kochinsky. 2014. A mapreduce algorithm to create contiguity weights for spatial analysis of big data. In Proceedings of the International Workshop on Analytics for Big Spatial Data (BigSpatial'14).

[17] Dan Lin, Elisa Bertino, Reynold Cheng, and Sunil Prabhakar. 2008. Position transformation: A location privacy protection method for moving objects. In Proceedings of the SIGSPATIAL ACM International Conference on Advances in Geographic Information Systems (GIS’08).

[18] Dan Lin, Elisa Bertino, Reynold Cheng, and Sunil Prabhakar. 2009. Location privacy in moving-object environments. Trans. Data Privacy 2, 1 (2009), 21-46.

[19] Anna Monreale, Dino Pedreschi, Ruggero G. Pensa, and Fabio Pinelli. 2014. Anonymity Preserving Sequential Pattern Mining, Vol. 22. 141-173. DOI : https://doi.org/10.1007/s10506-014-9154-6

[20] Mehmet Ercan Nergiz, Maurizio Atzori, Yucel Saygin, and Baris Guc. 2009. Towards trajectory anonymization a generalization-based approach. Trans. Data Priv. 2, 106 (2009), 47-75. DOI: https://doi.org/10.1145/1503402.1503413

[21] Ruggero G. Pensa, Anna Monreale, Fabio Pinelli, and Dino Pedreschi. 2008. Pattern-preserving k-anonymization of sequences and its application to mobility data mining. CEUR Workshop Proc. 397 (2008), 44-60. 
[22] Giorgos Poulis, Spiros Skiadopoulos, Grigorios Loukides, and Aris Gkoulala-Divanis. 2013. Select-organizeanonymize: A framework for trajectory data anonymization. Proceedings of the IEEE 13th International Conference on Data Mining Workshops (ICDMW'13). 867-874. DOI : https://doi.org/10.1109/ICDMW.2013.136

[23] Giorgos Poulis, Spiros Skiadopoulos, Grigorios Loukides, and Aris Gkoulalas. 2014. A priori-based algorithms for km-anonymizing trajectory data. Trans. Data Priv. 7, 2 (2014), 165-194.

[24] Giorgos Poulis, Spiros Skiadopoulos, Grigorios Loukides, and Aris Gkoulalas-Divanis. 2013. Distance-based kmanonymization of trajectory data. Proceedings of the IEEE International Conference on Mobile Data Management, Vol. 2. 57-62. DOI : https://doi.org/10.1109/MDM.2013.66

[25] Swaminathan Sankararaman, Pankaj Agarwal, Thomas Molhave, Jiangwei Pan, and Arnold Boedihardjo. 2013. Modeldriven matching and segmentation of trajectories. In Proceedings of the ACM International Conference on Advances in Geographic Information Systems (SIGSPATIAL'13).

[26] Shuo Shang, Lisi Chen, Zhewei Wei, Christian S. Jensen, Kai Zheng, and Panos Kalnis. 2017. Trajectory similarity join in spatial networks. Proc. Very Large Data Base Endow. 10, 11 (Aug. 2017), 1178-1189.

[27] Shuo Shang, Lisi Chen, Zhewei Wei, Christian S. Jensen, Kai Zheng, and Panos Kalnis. 2018. Parallel trajectory similarity joins in spatial networks. Very Large Data Base f. 27, 3 (June 2018), 395-420.

[28] Executive Summary. 2014. Cisco Visual Networking Index: Global Mobile Data Traffic Forecast Update, 2013-2018. Retrieved from http:/www.cisco.com/c/en/us/solutions/collateral/service-provider/visual-networking-index-vni/ white_paper_c11-520862.html.

[29] Weina Wang, Lei Ying, and Junshan Zhang. 2014. On the tradeoff between privacy and distortion in differential privacy. In Proceedings of the Special Interest Group on Knowledge Discovery and Data Mining (KDD’14). 517-525. Retrieved from http://arxiv.org/abs/1402.3757.

[30] Katrina Ward, Dan Lin, and Sanjay Madria. 2017. MELT: Mapreduce-based efficient large-scale trajectory anonymization. In Proceedings of the International Conference on Scientific \& Statistical Database Management (SSDBM'17).

[31] Roman Yarovoy, Francesco Bonchi, Laks V. S. Lakshmanan, and Wendy Hui Wang. 2009. Anonymizing moving objects: How to hide a MOB in a crowd? Proceedings of the 12th International Conference on Extending Database Technology Advances in Database Technology (EDBT'09). 72-83. DOI : https://doi.org/10.1145/1516360.1516370

[32] Weizhong Zhao, Huifang Ma, and Qing He. 2009. Parallel k-means clustering based on mapreduce. In Cloud Computing. Springer, Berlin, 674-679.

[33] Yu Zheng, Lizhu Zhang, Xing Xie, and Wei-Ying Ma. 2009. Mining interesting locations and travel sequences from GPS trajectories. In ACM Press. 791-800.

[34] Kathryn Zixkhur. 2013. Location-based Services. Retrieved from http://www.pewinternet.org/2013/09/12/locationbased-services.

Received May 2018; revised February 2019; accepted March 2019 